\title{
Die Übersetzung von Modalpartikeln als Indiz ihres Grammatikalisierungsgrades: die französischen Pendants von denn und eigentlich
}

\author{
Steven Schoonjans/Kurt Feyaerts (Leuven)
}

\begin{abstract}
In this paper, we consider the way modal particles are translated as an indication of their degree of grammaticalization. More in particular, the exemplar case studied here is the French translation of the German particles denn and eigentlich. Starting from a literary translation corpus, we will list five tendencies in the distribution of the translation equivalents which may indicate that denn has gone further already on the pathway of grammaticalization than has eigentlich. Thereby, we will focus on the meaning aspects of the grammaticalization process (persistence, bleaching, new meaning extension).
\end{abstract}

\section{$1 \quad$ Einleitung}

Die Übersetzung der deutschen Modalpartikeln ist ein Thema, das schon mehrfach in der Literatur angesprochen wurde. ${ }^{1}$ Auch der vorliegende Aufsatz ist dieser Forschungsfrage gewidmet. Dabei ist das Ziel keineswegs bloß eine Auflistung möglicher Pendants mit zusätzlich einer Erläuterung der Distributionsfaktoren. In diesem Aufsatz wird von der Annahme ausgegangen, dass die Übersetzungen einen wesentlichen Beitrag zur besseren Erkenntnis der Modalpartikeln liefern. Insbesondere wird an die Grammatikalisierungstheorie angeknüpft: es wird gezeigt, dass die Übersetzungen eher für als gegen die These sprechen, denn habe im Vergleich zu eigentlich schon einen weiteren Weg auf dem Grammatikalisierungskontinuum zurückgelegt. Die zugrunde liegende Annahme, nicht alle Modalpartikeln seien in demselben Maße schon Objekt der Grammatikalisierung gewesen, besteht schon länger. Ziel des vorliegenden Aufsatzes ist, diese Annahme noch zu verstärken, und zwar aus einem Blickwinkel, der dafür u. W. noch nicht angewandt wurde: ausgehend von Übersetzungen.

Der Aufsatz ist folgendermaßen aufgebaut: in den nächsten zwei Kapiteln wird der theoretische Rahmen (Grammatikalisierung und Übersetzung der Modalpartikeln) aufgebaut und wird das Forschungsobjekt (die Partikeln denn und eigentlich) vorgestellt, im vierten Kapitel wird auf die methodologischen Aspekte der Untersuchung eingegangen, und in den fünf anderen Kapiteln (5-9) wird jeweils ein Argument geliefert, das für die zentrale These dieses Aufsatzes spricht.

\section{Die Grammatikalisierung von Modalpartikeln}

Die Frage, ob bzw. inwieweit bei den Modalpartikeln von Grammatikalisierung die Rede sein kann, dürfte ein wenig kontrovers sein. Nach manchen Autoren handle es sich bei den Modalpartikeln tatsächlich um Produkte eines Grammatikalisierungsprozesses, der aber nicht ganz traditionell ablaufen dürfte (u. a. Autenrieth 2002, 2005; Abraham 2000; Diewald 2007; Wegener 2002). Molnár (2008) behauptet dagegen, die Abweichungen seien zu groß, damit

\footnotetext{
* Wir bedanken uns bei Herrn Peter Lauwers für seine nützlichen Hinweise während der Verarbeitung der Korpusdaten, sowie bei unserem Publikum auf der Tagung "40 Jahre Partikelforschung" (Bern, 11.-13.2.2009) für die interessanten Fragen und Anmerkungen. Daneben danken wir einem anonymen Gutachter von Linguistik Online für weitere wertvolle Hinweise.

${ }^{1}$ Für das Französische, das hier als Zielsprache verwendet wird, sollten u. a. Weydt (1969), Albrecht (1977), Burkhardt (1995), Métrich (1997, 2000), Feyrer (1998) und Waltereit (2006) erwähnt werden.
} 
noch von Grammatikalisierung die Rede sein könne. Aus Gründen, die hier nicht dargestellt werden können (vgl. dazu u. a. Auer/Günthner 2003: 16-22), wollen wir dennoch von Grammatikalisierung sprechen. Für die folgende Analyse ist diese Frage rein terminologisch und daher eher unproblematisch, da hier vor allem die Bedeutungsebenen der Grammatikalisierung in Betracht gezogen werden, und diese werden von Anna Molnár schon anerkannt.

Allerdings stellt sich dann die Frage, inwiefern Modalpartikeln eine eigene Bedeutung aufweisen. Im Allgemeinen (vgl. u. a. Thurmair 1989 und Diewald 2007) werden die Abtönungspartikeln zu den Synsemantika gerechnet, die zwar an und für sich keine lexikalische (oder propositionale) Bedeutung haben, was aber nicht heißen sollte, dass die Partikeln keinen Beitrag zur Bedeutung einer Äußerung liefern. Dieser Beitrag wäre aber auf der illokutiven Ebene zu situieren (vgl. die Diskussion, ob Modalpartikeln illokutionsindizierend oder -modifizierend wirken ${ }^{2}$, wo der Sprecher in seiner aktiven Rolle als Gesprächspartner mehr oder weniger sichtbar wird. So bringen Modalpartikeln u. a. zum Ausdruck, wie eine Aussage mit einer Vorgängeräußerung verknüpft wird, wie der Inhalt einer Vorgängeräußerung eingeschätzt wird usw.

Nach der Ansicht von Traugott (u. a. 1982 und 1995; vgl. auch De Mulder 2001: 18f.) ist die Bedeutungsentwicklung bei einem Grammatikalisierungsprozess als ein Prozess der Subjektivierung $\mathrm{zu}$ betrachten. Traugott umschreibt Grammatikalisierung als einen semantischen Prozess, der von der propositionalen über die textuelle bis hin zur expressiven bzw. subjektiven Ebene abläuft. Eine solche Entwicklung ist tatsächlich auch bei den Modalpartikeln nachweisbar. Auch wenn die Abgrenzung der drei Ebenen nicht immer unproblematisch ist, sei dieser Entwicklungsprozess hier für die beiden Partikeln denn und eigentlich näher illustriert. ${ }^{3}$

Die Modalpartikel denn findet ihren Ursprung im Konsekutivadverb dann (regional auch denn), das selber aus dem Temporaladverb entstanden ist. ${ }^{4}$ Mittels der Modalpartikel denn gibt der Sprecher an, dass ein bestimmter Sachverhalt (in der Vorgängeräußerung oder im außersprachlichen Kontext) für ihn unerwartet ist, und er bittet um irgendeine Erklärung oder Ergänzung bzw. eine Spezifizierung. Gelegentlich ist dabei auch ein gewisses Staune impliziert. Das ist eine expressive bzw. subjektive Verwendung, während das Temporaladverb deutlich als propositionales Element zu betrachten ist. Mit diesem expressiven Gebrauch hat der Grammatikalisierungsprozess allerdings noch nicht seinen Endpunkt erreicht, was daraus hervorgeht, dass denn sich zunehmend zu einem reinen Frageanzeiger ohne fest umreißbare Bedeutung weiterentwickelt (vgl. Thurmair 1989: 167-169).

(1) A: Am 18. Dezember kann ich nicht da sein; dann bin ich im Ausland. (Temporaladverb)

B: Dann verschieben wir den Termin eben um eine Woche. (Konsekutivadverb)

A: Arbeitest du denn am Weihnachtstag? (Modalpartikel)

\footnotetext{
2 Dazu u. a. Zifonun et al. (1997: 903).

3 Für die Wahl dieser beiden Partikeln sind vier Gründe zu nennen: erstens die Tatsache, dass diese beiden Partikeln gewisse Ähnlichkeiten aufweisen (u. a. ihr Auftreten in Fragen, sowohl Ergänzungs- als Entscheidungsfragen), was ein Vergleich nicht nur einfacher, sondern auch interessanter macht, zweitens die Tatsache, dass sie einfach kombiniert werden können (was ebenfalls eine interessante Ausbreitung des Vergleiches ermöglicht), drittens die Tatsache, dass es die beiden am häufigsten verwendeten Partikeln in Fragen sind (Hentschel/Weydt 1983: 272), und viertens die Feststellung, dass diese beiden Partikeln im Hinblick auf ihre Übersetzung noch nicht eingehend erforscht wurden. Die folgenden Bedeutungsbeschreibungen basieren auf den Darstellungen in allgemeineren Arbeiten wie Thurmair (1989) und Franck (1980).

${ }^{4}$ Es gibt noch eine andere Theorie zum Entstehen von denn, die von u. a. Molnár (2002) vertreten wird. Darauf wird in Kap. 6 noch weiter eingegangen, wenn von der Bedeutungspersistenz in denn die Rede ist. Es sei noch angemerkt, dass im vorliegenden Aufsatz von einem deutlichen Unterschied zwischen Modalpartikeln und Adverbien ausgegangen wird (ähnlich auch in Kap. 6 zwischen Diskurspartikeln und Adverbien). Dieser klare Unterschied wird manchmal kritisiert, und in der Tat ist die Grenzziehung nicht immer sehr einfach (vgl. auch Fussnote 12). Dass es diese Schwierigkeiten gibt, sollte u. E. nicht allzu sehr wundern, da die Grammatikalisierung hier eine wichtige Rolle spielt, und diese geht bekanntlich auf Kontinuen vor. Es sollte somit (sowohl auf deutscher als auch auf französischer Seite) eher von Kontinuen ausgegangen werden. Die Verwendung der Begriffe 'Modalpartikel' und 'Adverb' könnte also irrtümlicherweise den Eindruck einer diskreteren Einteilung wecken, dient aber der Übersichtlichkeit in der Darstellung, in der nur die Polen ("Quellform" und "Zielform") des Grammatikalisierungsprozesses genannt werden.
} 
Bei eigentlich liegt eine ähnliche Situation vor: die Modalpartikel ist aus dem homophonen Adverb mit textueller, oft kontrastierender Funktion entstanden. Mit der Modalpartikel wird aber angedeutet, dass zu einem anderen Thema bzw. zu einem anderen Themenaspekt übergegangen wird. Dies ist keine expressive Bedeutung im engsten Sinne des Wortes, weist aber eine subjektivere Funktion als die des Adverbs auf.

(2) [A redet schon seit fünf Minuten über seinen Bruder, dessen Namen er aber noch nicht erwähnt hat.]

B: Sag mal, wie heißt dein Bruder eigentlich? (Modalpartikel)

A: Ich nenne ihn meist Hansi, aber eigentlich heißt er Johann. (Adverb)

Wie oben schon angedeutet, werden im vorliegenden Beitrag vor allem die semantischen Aspekte der Grammatikalisierung in Betracht gezogen. An erster Stelle ist dabei an die nicht unabhängig voneinander wirksamen Mechanismen der Bedeutungspersistenz bzw. Bedeutungsverblassung zu denken. Gemeint ist, dass die ursprüngliche Bedeutung der Ausgangsform (zum Teil) verloren geht ("verblasst"), obwohl immer noch Aspekte der ursprünglichen Bedeutung nachweisbar bleiben (Persistenz). Etwa bei denn ist diese ursprüngliche Bedeutung die Angabe der Konsekutivität ${ }^{5}$, und wie sich zeigen wird, schwingt dieser Aspekt in manchen Gebrauchskontexten der Modalpartikel noch deutlich mit (Persistenz), während er in anderen nahezu völlig verschwunden ist (Verblassung).

Es ist auch möglich, dass im Laufe des Grammatikalisierungsprozesses neue Bedeutungsaspekte hinzugewonnen werden bzw. dass anderen Bedeutungsaspekten eine größere Rolle zufällt. Im Allgemeinen handelt es sich dann um Aspekte subjektiver Art auf der abstrakteren Ebene, auf der sich das grammatikalisierte Element bewegt (vgl. Heine 2003). Dies ist zum Beispiel bei eigentlich der Fall, wo der Aspekt des thematischen Übergangs kaum oder gar nicht ausgeprägt im Adverb nachweisbar ist. Im Folgenden wird auf diese Entwicklung mit dem Begriff "neue Bedeutungserweiterung" hingewiesen.

Auf noch andere Aspekte der Grammatikalisierung kann hier weiter nicht eingegangen werden, so etwa die Tatsache, dass ein grammatikalisiertes Element im Vergleich zu der ursprünglichen Form auch in anderen Kontexten verwendet werden kann. Ein Aspekt sei allerdings doch noch erwähnt, weil er in der weiteren Besprechung noch kurz in Betracht gezogen wird. Bei der Grammatikalisierung liegt häufig sogenannte Schichtung vor, das heißt, dass die "Quellform" noch neben der "Zielform" besteht. Dies trifft auf die vorliegende Studie zu: neben den Modalpartikeln werden auch die Adverbien dann (bzw. denn) und eigentlich noch als solche verwendet.

\section{Die Übersetzung von Modalpartikeln}

Typisch für (inter)subjektive Elemente wie Modalpartikeln ist, dass ihre Bedeutung erst im Gebrauchskontext voll zum Tragen kommt. Der Kontext muss dementsprechend bei der Übersetzungsarbeit uneingeschränkt in Betracht gezogen werden: "Wegen ihrer starken Kontextdeterminiertheit kann nur das Textganze an sich bzw. die Aussage im pragmatischkommunikativen Umfeld die übergeordnete Übersetzungseinheit für Aussagen mit MPn sein" (Feyrer 1998: 107). Infolge der starken Kontextdeterminiertheit der Partikelbedeutung sollte es nicht wundern, dass für die beiden hier untersuchten Partikeln jeweils verschiedene Übersetzungsäquivalente vorliegen (vgl. Feyrer 1998: 51; Lerot 2001: 126). Dabei kann es sich um lexikalische Pendants handeln, aber die Nuance kann genauso gut mittels bestimmter morphologischer Mittel (etwa einer bestimmten Verbform wie der 'conditionnel' oder der 'subjonctif'), syntaktischer Konstruktionen, intonatorischer Muster bzw. einer Kombination dieser Elemente ausgedrückt werden. Im vorliegenden Beitrag werden allerdings nur die rein lexikalischen Übersetzungen (sowie auch die Nichtübersetzung) in Betracht gezogen. Diese bilden aber weitaus die größte Gruppe (97,10\% der Belege von denn, 97,78\% der Belege von eigentlich).

\footnotetext{
5 Eigentlich ist die konsekutive Bedeutung nicht die ursprüngliche, da das Konsekutivadverb dann wie angesprochen selber aus einem Temporaladverb entstanden ist. In der folgenden Analyse wird aber jeweils nur ein Schritt zurück gemacht, zu dem direkten Vorfahr der Partikel (konsekutives dann bzw. adverbiales eigentlich), weil davon ausgegangen wird, dass Spuren älterer Phasen (temporales dann) in der Übersetzung kaum nachzuweisen sind.
} 


\section{$4 \quad$ Methodologische Aspekte der Untersuchung}

Da die Modalpartikeln ein typisch gesprochensprachliches Phänomen darstellen, sollte für diese Untersuchung von einem Korpus gesprochener Sprache ausgegangen werden. Da es aber u. W. keine Übersetzungskorpora gesprochenen Sprachgebrauchs gibt, musste eine Alternative gesucht werden: es wurden Texte verwendet, in denen gesprochene Sprache wiedergegeben wird. Wie manche andere Forscher, die sich schon mit der Übersetzung von Modalpartikeln beschäftigt haben (u. a. Feyrer 1998 und Rinas 2006), haben wir für ein literarisches Übersetzungskorpus optiert. Insgesamt handelt es sich um 21 deutsche literarische Werke (9 Prosatexte, 12 Theaterstücke), mit jeweils einer französischen Übersetzung. ${ }^{6}$ Dabei wurde einerseits darauf geachtet, dass die Übersetzer Muttersprachler des Französischen sind, damit sie bestens mit den subtilen Nuancen der französischen Ausdrücke vertraut sind (und so das geeignetste Pendant auswählen können). Andererseits wurde darauf geachtet, dass die verschiedenen Texte von unterschiedlichen Übersetzern ins Französische übertragen wurden, um mögliche Einflüsse persönlicher Präferenzen sowie einer eventuellen Nachlässigkeit oder 'Überübersetzung' (vgl. Métrich 1997: 149) zu vermeiden. Außerdem wurden Belege, in denen die französische Übersetzung allzu sehr vom deutschen Original abweicht (zum Beispiel durch eine allzu eigenständige Interpretation des Übersetzers oder weil ein Satz bzw. ein ganzer Auszug zwar dieselbe Gesamtbedeutung aber eine ganz andere Form hatte), außer Betracht gelassen. Insgesamt konnten so 518 "verwendbare" Belege von denn und 45 von eigentlich aufgefunden werden. ${ }^{7}$

\section{$5 \quad$ Das allgemeine Bild}

Nach diesen einführenden Kapiteln können jetzt die Ergebnisse besprochen werden. Ziel ist dabei nicht, auf alle im Korpus vorliegenden Übersetzungen einzugehen. Vielmehr wird versucht, in den folgenden Kapiteln (5-9) fünf Tendenzen zu identifizieren, die mit der Ausgangsthese des vorliegenden Aufsatzes im Einklang sind, nämlich dass der Grammatikalisierungsprozess bei denn weiter vorangeschritten ist als bei eigentlich. Es soll also gezeigt werden, dass eigentlich noch eine stärkere und deutlicher abgrenzbare Bedeutung aufweist als denn, was mit einer größeren Persistenz bei eigentlich und somit einem geringeren Grammatikalisierungsgrad einhergeht. Zunächst wird in diesem Kapitel vom allgemeinen Bild ausgegangen, dann werden die drei semantischen Aspekte der Grammatikalisierung (Bedeutungsverblassung, Bedeutungspersistenz, neue Bedeutungserweiterung) in je einem Kapitel (6-8) behandelt, und zum Schluss wird im Kapitel 9 noch kurz etwas über die Kombination denn eigentlich ausgesagt.

Wenn die Ergebnisse grafisch wiedergegeben werden, ergibt sich folgendes Bild:

\footnotetext{
${ }^{6}$ Die Liste der verwendeten Texte ist der Bibliographie zu entnehmen. Jedesmal steht vor der Referenz ein dreistelliges Kürzel. Dies wird im Folgenden verwendet, um auf die Texte zu verweisen. $\mathrm{Zu}$ der Liste sei noch erwähnt, dass bei ele nicht die Opernfassung, sondern die ältere Dramenfassung verwendet wurde (um möglichen Einfluss des Rhythmus auf Syntax und Wortwahl zu vermeiden), und dass die beiden anderen Texte aus diesem Band (Der Rosenkavalier und Ariadne auf Naxos) nicht in das Korpus aufgenommen wurden.

7 Es handelt sich nur um Einzelpartikeln. Belege in Kombinationen wie denn blo $\beta$ werden nicht weiter berücksichtigt. Die einzige Ausnahme bildet die Kombination denn eigentlich, die in Kap. 8 kurz behandelt wird, und die insgesamt zehnmal im Korpus vorliegt, hier aber ebenfalls nicht mitgezählt wurde.
} 


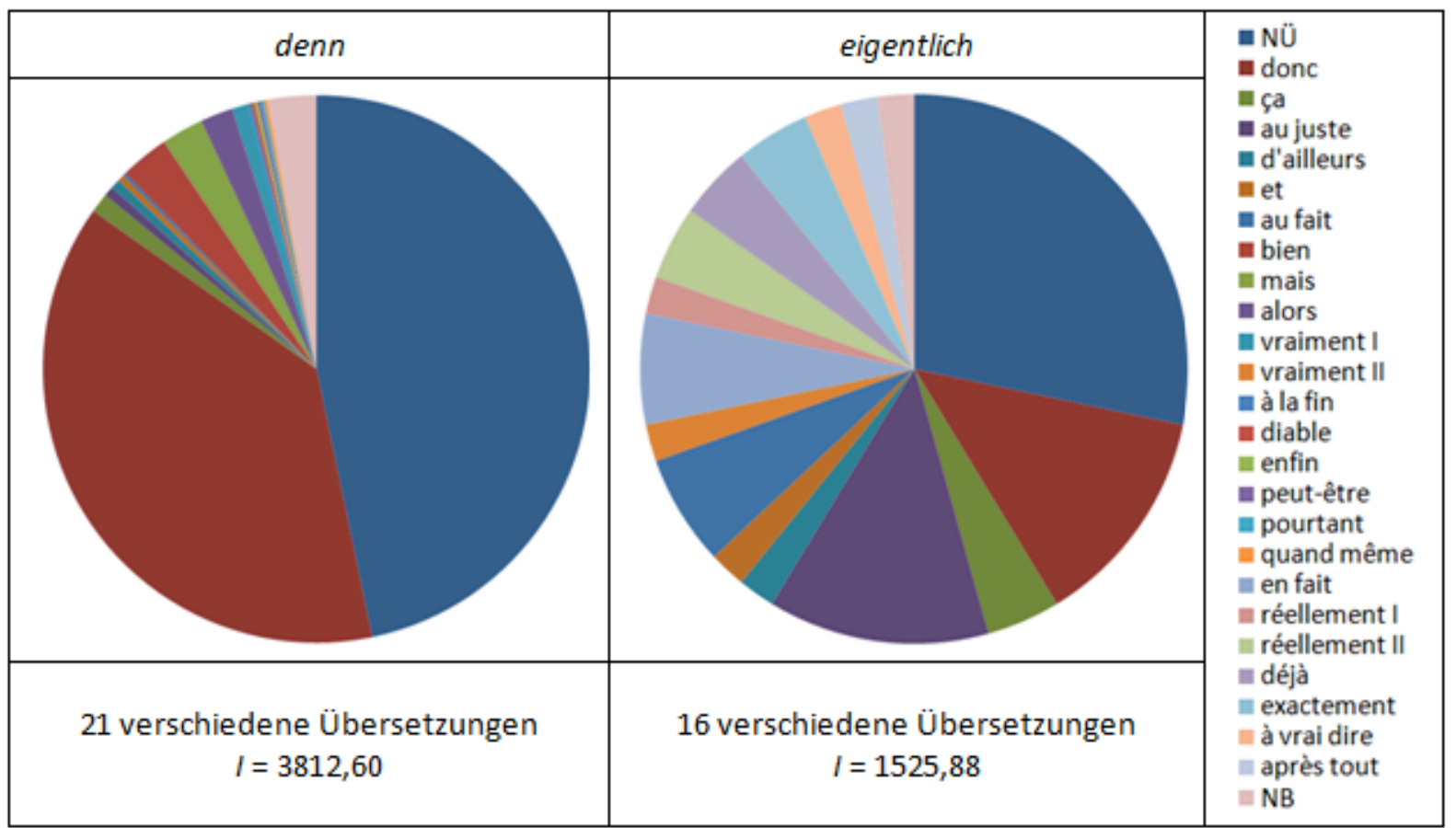

Abb. 1: Die Distribution der vorliegenden Übersetzungen (NÜ = Nichtübersetzung; NB = nicht betrachtet, d. h. morphologisch o. dgl.) ${ }^{8}$

Wenn man die Diagramme nur oberflächlich betrachtet und dabei die Legende kurz beiseite lässt, könnte man meinen, denn habe eine deutlich abgrenzbare Bedeutung, während das bei eigentlich viel weniger der Fall zu sein scheint. Dieser Eindruck wird wohl dadurch bestimmt, dass bei denn zwei deutlich dominierende Übersetzungen vorliegen, was bei eigentlich nicht in demselben Maße der Fall ist. Dies zeigt sich auch in den jeweils unten angegebenen 'internen Uniformitätswerten' (I-Werte, vgl. Geeraerts et al. 1999), die ein Maß für die Homogenität einer Datensammlung sind: je gleichartiger die Daten sind, umso höher ist der IWert. Es zeigt sich, dass der I-Wert bei denn mehr als doppelt so hoch ist wie bei eigentlich.

Diese These wird noch attraktiver, wenn man weiß, dass die beiden dominanten Übersetzungen semantisch ähnlich sind. Dennoch darf diese Schlussfolgerung nicht gezogen werden, denn wie ein Blick auf die Legende verrät, sind die beiden dominanten Übersetzungen bei denn gerade jene zwei mit der vagsten Bedeutung: die Nichtübersetzung und donc. Auf diese beiden Pendants wird im folgenden Kapitel tiefer eingegangen, wo vom Einfluss der Bedeutungsverblassung die Rede ist. Da es sich aber um zwei relativ bzw. absolut bedeutungsleere Elemente handelt, kann daraus nicht geschlussfolgert werden, denn habe eine deutlicher abgrenzbare Bedeutung als eigentlich.

Die Daten in Abbildung 1 zeigen ebenfalls, dass bei denn eine größere Anzahl unterschiedlicher Übersetzungen vorliegt als bei eigentlich. Allerdings dürfen die Daten nicht einfach ausgehend von der Zahl der Übersetzungsäquivalente interpretiert werden, und zwar aus drei Gründen. Erstens enthält das Korpus für eigentlich weniger Belege, und ist somit weniger repräsentativ was die selteneren Pendants betrifft (die wichtigsten Tendenzen, die im Folgenden besprochen werden, treten aber schon deutlich hervor). Zweitens muss erwähnt werden, dass eine reichere Variation auf der Ebene der vorgefundenen Übersetzungsäquivalente nicht unbedingt auf eine heterogenere Datensammlung schließen lässt. Dies zeigt sich auch in den Daten, wo bei denn die interne Uniformität größer ist, obwohl es mehr unterschiedliche Übersetzungen gibt. Dies hat damit zu tun, dass manche Pendants nur sehr selten auftreten, und (wie z. B. pourtant) nur in bestimmten Kontexten möglich sind, etwa wenn von der Satzstruktur her etwas freier übersetzt wird. Drittens sind manche (vor allem lexikalische) Pendants synonym, oder wenigstens dermaßen bedeutungsgleich, dass sie gemeinsam als eine Übersetzungsweise betrachtet werden können. Das ist etwa der Fall mit

\footnotetext{
${ }^{8}$ Der Unterschied zwischen réellement I und II bzw. vraiment I und II wird in den folgenden Kapiteln weiter kommentiert. Bei der Zählung wurden die verschiedenen nichtbetrachteten Übersetzungen schon einzeln gezählt.
} 
réellement II und vraiment II bei eigentlich (siehe auch die unten zu besprechende große "Persistenzgruppe" bei dieser Partikel (Kap. 6).

Immerhin bleibt es so, dass denn eine größere Verschiedenheit unter den Übersetzungsäquivalenten aufweist als eigentlich. Dies hängt zum Teil mit der sogenannten neuen Bedeutungserweiterung zusammen, zu der unten noch zu zeigen sein wird, dass sie bei denn eine größere Rolle spielt als bei eigentlich. Daneben kommt diese größere Vielfalt vor allem bei den weniger stark vertretenen Übersetzungen zum Ausdruck. Dabei muss allerdings dem schon erwähnten Umstand Rechnung getragen werden, dass auf dieser Ebene das Korpus für eigentlich etwas weniger repräsentativ sein dürfte.

Allerdings kann aus dem bisher Gesagten die interessante These abgeleitet werden, dass die Daten für denn zugleich (aber auf unterschiedlichen Ebenen) homogener und heterogener sind als die Daten für eigentlich (heterogener, weil mehr unterschiedliche Übersetzungen vorliegen, aber zugleich auch homogener, weil eine stärkere Dominanz der wichtigsten Pendants vorliegt). Daneben dürfte auch deutlich sein, dass für die hiesige Argumentation vor allem der Aspekt der größeren Vielfalt von Bedeutung ist, da der sich auf die aussagekräftigeren Übersetzungspendants bezieht, und somit ein Indiz dafür ist dass es bei denn schwieriger ist, eine genaue Bedeutung abzugrenzen. Die Homogenität dagegen ist eher mit dem Prozess der Bedeutungsverblassung zu verknüpfen, der anschließend im Kapitel 6 zum Thema wird.

Zum Schluss sei noch angemerkt, dass die Grammatikalisierung nicht als einzige Erklärung der Vielfalt der Übersetzungen der beiden Partikeln gelten kann. Nur Bedeutungspersistenz, Bedeutungsverblassung und neue Bedeutungserweiterung reichen nicht aus, um diese ganze Verschiedenheit zu deuten. Ein ganz wichtiges anderes Element (das außerdem nah mit neuer Bedeutungserweiterung verknüpft ist) wurde oben schon angesprochen: die genaue Bedeutung von Modalpartikeln ist in hohem Maße kontextabhängig, und je nach Kontext können andere Implikaturen realisiert werden, die in der Übersetzung explizit ausgedrückt werden können. Daneben muss berücksichtigt werden, dass es sich immerhin um zwei unterschiedliche Sprachen handelt. Man muss also dem Umstand Rechnung tragen, dass der Wortschatz dieser beiden Sprachen nicht ganz übereinstimmt, weder im Umfang noch in der Spezifizität und Vielfalt der Wörter. Mit anderen Worten: man sollte die Anzahl der Ausdrücke in einem Bedeutungsfeld, die Allgemeinheit ihrer Bedeutung und die Subtilität der Unterschiede auf der Bedeutungsebene in Betracht nehmen (vgl. die oben angesprochene Quasisynonymie einiger Übersetzungsäquivalente). In dieser Hinsicht ist auch Roman Jakobsons These relevant, dass "[1]anguages differ essentially in what they must convey and not in what they may convey" (1959: 236, seine Hervorhebungen). Es ist im Rahmen des vorliegenden Aufsatzes nicht möglich, dieser Frage weiter nachzugehen; es dürfte nur deutlich sein, dass mit Grammatikalisierung nicht die ganze Vielfalt der Übersetzungen erklärt werden kann, obwohl es ein interessanter Ansatz für die Interpretation der vorliegenden Ergebnisse ist.

\section{Die Rolle der Bedeutungsverblassung}

Ziel des vorliegenden Beitrags ist, zu zeigen, dass denn einen höheren Grammatikalisierungsgrad aufweist als eigentlich. Dabei ist eine fortgeschrittene Bedeutungsverblassung typisch für eine fortgeschrittene Grammatikalisierung. Dies zeigt sich in den Übersetzungen, und zwar in solchen, die eine eher vage Bedeutung aufweisen. Es wurde schon darauf hingewiesen, dass bei denn zwei solche Übersetzungen stark vertreten sind: donc und die Nichtübersetzung. Da diese auch bei eigentlich in nicht allzu geringem Maße vertreten sind, eignen sie sich gut für einen Vergleich.

Bevor tiefer darauf eingegangen wird, seien aber noch einige Bemerkungen zur Nichtübersetzung gemacht, weil diese sich manchmal auch durch andere Faktoren als die Bedeutungsverblassung erklären ließe..$^{9}$ Ein erster Aspekt ist die Intonation, zu der schon angedeutet wurde, dass sie bei den subtilen Bedeutungsnuancen der Modalpartikeln nicht unwichtig ist. Häufig hat sie eine unterstützende Funktion, aber in Sprachen wie dem Französischen kann

\footnotetext{
${ }^{9}$ Die meisten dieser Faktoren wurden schon von anderen Forschern identifiziert, etwa Métrich (1997, 2000), Waltereit (2006) und Zimmermann (1981).
} 
sie manchmal auch alleine diese Nuancen zum Ausdruck bringen. Das führt allerdings zum methodologischen Problem, dass in unserem Korpus keine Intonation vorliegt. Zwar liefern die Zeichensetzung, der Satzbau, der Kontext, bestimmte Beschreibungen im Text sowie bei den Dramen auch die Regieanweisungen einige brauchbare Hinweise zur Prosodie, aber eine genaue Bestimmung der bzw. einer möglichen Intonation ist ausgeschlossen. Es ist somit nicht völlig auszuschließen, dass ein etwas größerer Anteil der Partikeln hier als "nichtübersetzt" bezeichnet wird, als bei einem Korpus mit Intonation der Fall gewesen wäre, wobei allerdings ein paralleles Übersetzungskorpus frei gesprochener Sprache weiterhin ein Forschungsdesiderat bleibt.

Daneben kann der Anlass zum Nichtübersetzen auch im sprachlichen Kontext gegeben sein. So kann im gleichen Satz bzw. in derselben Äußerung noch ein anderes Element vorhanden sein, das mit der Partikel bedeutungsgleich oder bedeutungsähnlich ist, etwa das zum Kuckuck in (3). Wenn dieses Element übersetzt wird, kann die Partikel selber in der Übersetzung ausfallen. Übrigens ist es so, dass die Übersetzung dieses Elementes im Allgemeinen auch als Pendant der Partikel selber verwendet werden kann, wie sich in Beispiel (4) für das Pendant von zum Kuckuck, diable, zeigt:

(3) Wer, zum Kuckuck, ist denn das? (mjt 141)

Qui diable est cet individu ? (134)

(4) Warum kommst du denn erst jetzt auf die Idee, nach Tabletten zu fragen? (hit 155)

Pourquoi diable est-ce aujourd'hui seulement qu'il te vient à l'esprit de demander des pastilles? (148)

Eine ähnliche Situation liegt vor, wenn sich die Nuance der Partikel auf mehrere aufeinanderfolgende Sätze bezieht. Im Allgemeinen wird dann im Deutschen die Partikel jedesmal wiederholt, während im Französischen die Nuance oft (7 Fälle von 14 im Korpus) nur einmal expliziert wird. Meist ist es dann die erste Partikel, die in der Übersetzung ein lexikalisches Pendant aufweist:

(5) Sind Sie denn blind? Sehen Sie denn nicht, wie er dasitzt und schweigt und uns reden lässt? (mjt 135)

Seriez-vous donc aveugle ? Ne voyez-vous pas le baron qui se tait et nous laisse parler ? (128)

Das sind drei Faktoren (Intonation, bedeutungsähnliches Element, Wiederholung der Partikel), die schon einen Teil der nichtübersetzten Fälle deuten (können). Allerdings lassen sich bei Weitem nicht alle Fälle so erklären. Außerdem kann das Auftreten von donc auf keinen Fall einem dieser drei Faktoren zugeschrieben werden. Es muss also ein anderer Grund gesucht werden. Oben wurde schon angedeutet, dass Texte von verschiedenen Übersetzern verwendet wurden, damit eine idiosynkratische Vernachlässigung der Partikeln bei einzelnen Übersetzern die Ergebnisse nicht allzu sehr beeinflussen kann. Außerdem ist es so, dass in allen französischen Texten ein beträchtlicher Anteil der Partikeln schon ein Pendant hat. Nachlässigkeit seitens der Übersetzer lässt sich daher schon ausschließen. Sie könnte außerdem ohnehin die Belege von donc nicht erklären, d. h. höchstens in dem Maße, als dann nur ein bedeutungsvages Passepartout eingesetzt würde.

Es gibt noch ein zusätzliches Argument, um die Nachlässigkeitsthese zu verwerfen: sie erklärt nicht, warum diese beiden Übersetzungen bei denn viel häufiger auftreten als bei eigentlich (46,91\% NÜ und 38,22\% donc bei denn bzw. 31,11\% und 13,33\% bei eigentlich). Eine Kontrolle im Korpus hat übrigens ergeben, dass auch bei anderen Partikeln, die u. E. eine stärkere Bedeutung haben als denn (u. a. eben und schon), weniger oft auf diese französische Äquivalente zurückgegriffen wird. Das führt zu der Vermutung, dass der Distributionsunterschied mit den deutschen Partikeln selber zusammenhängt.

Ein Ansatz für eine Erklärung kann auf Weydt (1969) und Liefländer-Koistinen (1988) basiert werden. Sie geben an, dass in literarischen Texten häufiger Modalpartikeln verwendet werden als in der Alltagssprache, etwa um die Mündlichkeit der Dialoge deutlicher zu simulieren. Es würde sich da dann eher um bloße "Füllwörter" als um "bedeutungstragende" Partikeln handeln. In dem Fall sollte es nicht allzu sehr wundern, dass so manche Partikel beim Übersetzen verschwindet. Nur stellt sich dann wieder die Frage: wieso ist das bei denn 
häufiger als bei eigentlich der Fall? Vielleicht weil denn sich besser zur Verwendung als "Füllwort" eignet, oder aber weil diese Analyse auch keine (oder wenigstens keine vollständige und endgültige) Erklärung liefert.

Eine Analyse im Rahmen der Grammatikalisierungstheorie könnte hier Aufschluss bringen. U. E. ist die größere Zahl der in der Übersetzung ausgelassenen bzw. mit donc übersetzten Belege von denn dadurch zu erklären, dass denn schon eine größere Distanz auf dem Grammatikalisierungsweg zurückgelegt hat, und daher schon stärker dem Prozess der Bedeutungsverblassung verfallen ist. Für eigentlich gilt dies in geringerem Maße, und daher sind diese beiden Übersetzungsweisen bei dieser Partikel seltener. Übrigens wäre diese Analyse auch die Erklärung für die These, denn sei ein besseres "Füllwort" als eigentlich.

Zum Schluss dieses Kapitels sei die dargelegte Situation bei denn und eigentlich noch kurz anhand einige Beispiele verdeutlicht:

(6a) Wo ist das denn passiert? (hit 254)

Où cela s'est-il passé ? (241)

(6b) Kann man denn vergehen, lebend, wie ein faules Aas? (ele 118)

Est-ce qu'on peut, vivant, pourrir comme une charogne qui se décompose ? (119)

(6c) Was denn für Papiere? (aut 174)

Quels papiers ? (175)

(7a) Gehören die Hebräer denn neuerdings zur Una Sancta? (ste 367)

Les Hébreux font-ils donc partie maintenant de l'Una Sancta? (248)

(7b) Was machst du denn in dieser finsteren Gegend? (hit 95)

Que fais-tu donc dans ces tristes parages ? (91)

(7c) Wieso denn Ingolstadt? (mlu 70)

Pourquoi donc Ingolstadt ? (71)

(8a) Kennen Sie eigentlich den Untersuchungsrichter? (pro 50)

Connaissez-vous le juge d'instruction ? (118)

(8b) Wann kommt Frau Römer eigentlich aus der Kur zurück? (hit 89)

Quand donc Mme Römer va-t-elle rentrer de sa cure ? (85)

Wie sich in diesen Beispielen zeigt, sind diese Äquivalente nicht nur sehr vage von der Bedeutung her; daneben (und vielleicht auch zum Teil daher) unterliegen sie auch keinen wichtigen Einschränkungen, was ihr Auftreten betrifft. Gemeint ist damit, dass sie sowohl in Entscheidungs- als auch in Ergänzungsfragen verwendet werden können, und dass auch zum Beispiel das Vorliegen bzw. das Fehlen eines Verbs keinen Einfluss auf die Distribution dieser Übersetzungsweisen hat.

Eine Anmerkung sollte vielleicht noch gemacht werden. Dass die Nichtübersetzung einen leeren Semantismus mit sich trägt, dürfte einleuchten. Auf donc trifft das aber nicht zu: dieses Wort liefert einen illokutiven Beitrag, und zwar deutet es eine illokutive Verstärkung an (vgl. Mosegaard Hansen 1997: 168). Von der Bedeutung her situiert donc sich somit mehr oder weniger auf derselben Ebene wie die Modalpartikeln ${ }^{10}$, wobei illokutive Verstärkung allerdings als eine eher subjektive und nicht sehr spezifische Bedeutung zu betrachten ist. Allerdings ist es deutlich eine schwächere Bedeutung als die der Modalpartikel eigentlich und einiger anderer Übersetzungsäquivalente der Partikeln (vgl. u. a. Kap. 6). Dasselbe gilt auch für ein selteneres Pendant von denn (mit nur einem Beleg im Korpus), das ebenfalls schon erwähnt wurde: diable. Daher werden solche Übersetzungen auch zu denen gezählt, die mit Bedeutungsverblassung zu verknüpfen sind. Diese Hinzufügung von Elementen wie diable verändert aber nicht das Kernelement dieses Kapitels, dass bei denn häufiger für diese bedeutungsvagen bzw. bedeutungslosen Äquivalente optiert wird als bei eigentlich.

\footnotetext{
10 Mosegaard Hansen (1998: 42) betrachtet donc als eine Modalpartikel des Französischen.
} 


\section{$7 \quad$ Die Rolle der Bedeutungspersistenz}

Wo im vorigen Kapitel auf den Prozess der Bedeutungsverblassung eingegangen wurde, ist hier dessen Gegenstück im Grammatikalisierungsprozess, die Bedeutungspersistenz, zu besprechen. Gemeint ist die semantische Entwicklung, bei der in einer jüngeren, (teils) grammatikalisierten Bedeutung noch etwas von der ursprünglichen Bedeutung mitschwingt. Dieses Phänomen ist bei beiden hier präsentierten Partikeln deutlich nachweisbar, nicht zuletzt auch in deren französischen Übersetzungen. Im Einklang mit der Ausgangsthese der weiteren Grammatikalisierung von denn wäre dann die Feststellung, dass die Persistenz stärker ist bei eigentlich und somit einen größeren Einfluss auf die Übersetzung dieser Partikel hat.

Zunächst aber zu denn. Wie schon angedeutet wurde, ist diese Modalpartikel aus dem Konsekutivadverb dann (regional auch denn) entstanden. Es ist gerade diese Konsekutivbedeutung, die auch bei der Modalpartikel noch mitschwingen kann. Es handelt sich also um Nuancen wie 'wer/wo/was... (dann), wenn nicht X?' oder 'wer/wo/was... (dann), wenn das so ist?' in Ergänzungsfragen bzw. deren Pendant ohne Fragewort in Entscheidungsfragen:

(9) ERNST Ich weiß gar nicht, was schreiben.

OTTO Warst du denn nicht da, als uns Affenschmalz die Disposition gab? (frü 54)

ERNST Je ne sais pas du tout quoi mettre.

OTTO Alors tu n'étais pas là quand Singegraisse nous a donné l'énoncé ? (74) ${ }^{11}$

Dieses Beispiel weist mit dem Adverb alors gleich eine typische Übersetzung auf. Es sollte nicht allzu sehr wundern, dass dieses Adverb hier als Übersetzung vorliegt, da es als französisches Pendant des deutschen Adverbs dann gerade diese Konsekutivbedeutung aufweist. ${ }^{13}$ Insgesamt ist alors im Korpus zehnmal als Übersetzung von denn belegt. Sieben dieser Belege sind dem gerade illustrierten Fall zuzuordnen, der hier das Label 'Adverb' trägt. Dieses Label ist nicht unwichtig, da noch drei andere Belege von alors vorliegen, die nicht als Adverb einzustufen sind, sondern als Diskurspartikel:

(10) [Elvira erzählt einem Freund, den sie lange nicht gesehen hat, ihre Lebensgeschichte, und geht nachher weiter mit der Frage, wie es ihm mittlerweile vergangen ist:]

Naja... Wie ists [sic] denn so dort auf dem Land? (bvn 198)

Enfin... Alors, c'est comment, la campagne ? (199)

Es sei dazu allerdings angemerkt, dass die Einstufung auch auf französischer Seite nicht immer sehr einfach ist:

(11) BARBLIN ANDRI

BARBELINE

ANDRI
Andri - du bist keiner!

Warum willst du mich denn verstecken? (and 92)

Mais, Andri, tu n'es pas juif.

Alors, pourquoi veux-tu me cacher? (160)

\footnotetext{
${ }^{11}$ Es sei hier auf eine Schwierigkeit hingewiesen, die in der Literatur schon mehrfach angesprochen wurde. Es ist nämlich (vor allem in Ergänzungsfragen) nicht immer gleich deutlich, ob denn als Adverb oder als Modalpartikel eingestuft werden sollte. Im Falle der unbetonten Form dürfte es sich wohl um die Modalpartikel handeln, aber im Falle der betonten Form besteht auch in der Forschungsliteratur keine Einigkeit darüber (vgl. u. a. Wegener 2002: 390-392). Wir neigen allerdings eher dazu, sie zu den Adverbien zu zählen. Außerdem fehlt die Intonation im Korpus, und somit kann die Betonung nicht bestimmt werden. Dies erschwert die genaue Einstufung von Fällen wie (i), die u. E. aber eher den betonten Fällen zuzurechnen sind:
}

(i) KLYTÄMNESTRA Die Bräuche sag! Wie brächt ichs dar? Ich selber muss -

ELEKTRA Nein. Diesmal gehst du nicht auf die Jagd mit Netz und Beil.

KLYTÄMNESTRA Wer denn? Wer bringt es dar? (ele 122)

CLYTEMNESTRE Dis-moi les rites ! Comment sacrifier ? dois-je moi-même...

ELECTRE Non. Cette fois-ci ce n'est pas toi qui partiras en chasse avec un filet et une hache.

CLYTEMNESTRE Qui alors? qui accomplira le sacrifice? (123) 
Da aber deutlich eine konsekutive Nuance vorliegt (oder wenigstens vorliegen kann), sind solche Fälle (zwei sind es insgesamt) zur ersteren Gruppe gezählt worden.

Der Unterschied zwischen Adverb und Diskurspartikel ist hier nicht unwichtig, da ein anderes Verhältnis zur Partikel denn vorliegt. Das Adverb alors hat eine deutlich konsekutive Bedeutung, während die Diskurspartikel eher eine rein konnektive Funktion hat (vgl. die <KONNEX>-Funktion, die Thurmair 1989 mehreren Modalpartikeln zuweist), eher als eine ausgesprochene propositionale Bedeutung. Für die hiesige Argumentation ist es also vor allem das Adverb, das berücksichtigt werden soll, da es von einer größeren Bedeutungspersistenz auf deutscher Seite zeugt.

Um diese Besprechung der Persistenz in denn abzuschließen, sei noch kurz auf die bereits angesprochene Analyse von u. a. Molnár (2002) eingegangen. Sie geht davon aus, dass die Modalpartikel denn nicht aus dem Konsekutivadverb, sondern aus der Konjunktion denn entstanden ist. In diesem Fall wäre dann die konsekutive Nuance nachher als optionaler Bedeutungsaspekt der Modalpartikel hinzugefügt worden. Diese Analyse ist u. E. weniger plausibel als die hier dargelegte. Wenn sie aber richtig wäre, würde das die Argumentation nicht problematisieren. Die gerade besprochenen Belege mit konsekutivem alors würden dann eher dem nächsten Kapitel (zur neuen Bedeutungserweiterung) zugeordnet werden, aber wie sich noch zeigen wird, wäre das eher noch eine Verstärkung als eine Abschwächung der Argumente.

Wenn wir uns wieder der Bedeutungspersistenz zuwenden, so zeigt sich das Adverb alors als das einzige Übersetzungsäquivalent von denn, das wirklich mit Persistenz zu verknüpfen ist. Mit 7 Belegen aus 518 ist sie aber nicht gerade stark vertreten. Dies sollte nicht allzu sehr wundern, da die Zahl der Belege der Modalpartikel im Korpus, in denen die konsekutive Nuance noch deutlich mitschwingt (bzw. mitschwingen könnte), ohnehin nicht sehr hoch ist.

Ein ganz anderes und komplexeres Bild zeigt sich bei eigentlich, wo nicht von einem einzelnen Pendant die Rede sein sollte, sondern von einer ganzen Gruppe von Pendants, die alle von einer gewissen Bedeutungspersistenz zeugen, und die gemeinsam 33,33\% der Belege im Korpus ausmachen. In dieser Beobachtung sieht sich die Hypothese dieses Kapitels bestätigt, dass bei eigentlich die Bedeutungspersistenz eine größere Rolle für die Übersetzung spielt.

Es wurde oben schon erwähnt, dass die Modalpartikel eigentlich aus dem homophonen Adverb entstanden ist. Dies hat eine Bedeutung wie 'im Grunde genommen, ursprünglich, wirklich'. Diese Bedeutung kann in der Modalpartikel noch durchschimmern, und oft ist das (in stärkerem oder weniger starkem Maße) der Fall. Dies zeigt sich auch darin, dass manchmal nicht gleich auf den ersten Blick deutlich ist, ob ein Beleg als Adverb oder als Modalpartikel eingestuft werden sollte.

Typische Übersetzungspendants für die Belege der Modalpartikel, die von einer starken Bedeutungspersistenz zeugen, sind Elemente wie en fait, au juste, exactement, réellement II und vraiment II:

(12) Hier gibt es sicher keinen Satellitenempfang. Wo sind wir eigentlich? (aut 118) Il n'y a certainement pas de chaînes par satellite. Où on est, en fait ? (119)

(13) Möchte doch wissen, wozu wir eigentlich auf der Welt sind! (frü 11) J'aimerais pourtant savoir pourquoi au juste nous sommes dans ce monde ? (19)

(14) Um was handelt es sich eigentlich? (mjt 78)

De quoi s'agit-il, exactement? (75)

(15) So erinnere ich mich an ein Gespräch mit einem Schulkameraden, der mich fragte, für was ich mich eigentlich interessierte. (mar 36)

Ainsi je me rappelle une conversation avec un condisciple qui voulait savoir à quoi je m'intéressais vraiment. (44)

(16) Was wollen Sie eigentlich von mir? (ste 326)

Que voulez-vous réellement de moi ? (221) 
Mit diesen Elementen gibt der Sprecher an, dass er nähere bzw. genauere Informationen zu einem ihm bekannten Sachverhalt haben möchte (vgl. dazu u. a. Albrecht 1976: 70f.), was mit der oben erwähnten Grundbedeutung des Adverbs eigentlich im Einklang ist. Demgemäß sind diese Pendants eigentlich als Übersetzungen des Adverbs eigentlich zu betrachten, und zeugen somit von einer deutlichen Bedeutungspersistenz in der Modalpartikel eigentlich. ${ }^{12}$

Zum Schluss dieses Kapitels sei noch erklärt, warum von réellement II und vraiment II die Rede ist. Dies hat damit zu tun, dass die beiden Adverbien noch eine andere Verwendung (réellement $\mathrm{I}$ bzw. vraiment $\mathrm{I}^{13}$ ) haben, die auch im Korpus belegt ist, und auf die im folgenden Kapitel eingegangen wird. Es handelt sich um einen Ausdruck des Unglaubens, wie sich auch in den Beispielen des folgenden Kapitels zeigen wird.

Zunächst aber sei das Obige noch einmal auf den Punkt gebracht. Es hat sich gezeigt, dass sowohl bei denn als auch bei eigentlich Übersetzungspendants vorliegen, deren Vorkommen sich durch einen Prozess der Bedeutungspersistenz auf deutscher Seite erklären lässt. Auffällig ist dabei, dass diese Pendants bei eigentlich 33,33\% der Belege ausmachen, während es bei denn nur um 1,35\% geht. Dies ist im Einklang mit der zentralen These des vorliegenden Aufsatzes: wenn denn (schon) weiter grammatikalisiert ist als eigentlich, ist bei ersterer Partikel ein geringerer Einfluss der Bedeutungspersistenz zu erwarten, und das ist gerade, was sich in den Übersetzungen zeigt.

\section{Die Rolle der neuen Bedeutungserweiterung}

In den beiden vorigen Kapiteln wurden schon die Prozesse der Bedeutungspersistenz und der Bedeutungsverblassung besprochen. Im Grammatikalisierungsprozess ist allerdings noch ein dritter semantischer Entwicklungsaspekt zu unterscheiden, und zwar die sogenannte "neue Bedeutungserweiterung", auf die hier weiter einzugehen ist. Heine (2003: 591f.) weist schon darauf hin, dass ein Element im Laufe des Grammatikalisierungsprozesses neue Bedeutungsaspekte hinzugewinnen kann. Das gilt auch für die Modalpartikeln. Die Bedeutungsverblassung ermöglicht eine Verwendung in einer breiteren Skala von Kontexten, mit denen bestimmte zuvor nicht vorhandene Bedeutungsnuancen einhergehen können. Diese Nuancen müssen dann nicht expliziert werden, sondern können als eine Art Implikaturen allmählich in die Partikelbedeutung aufgenommen werden. ${ }^{14}$ Im Korpus liegen einige Belege vor, deren Übersetzung auf ein solches zusätzliches Bedeutungselement schließen lässt. Zu erwarten ist einerseits, dass dieses Phänomen bei denn häufiger nachweisbar ist, und andererseits, dass diese Partikel eine größere Verschiedenheit an neuen Nuancen aufweist. Der Grund ist, dass eigentlich infolge der ausgesprochenen Bedeutungspersistenz noch eine zu starke Bedeutung hat, während denn infolge seiner weit fortgeschrittenen Bedeutungsverblassung eine dermaßen schwache Eigenbedeutung aufweist, dass diese Partikel sich leichter in solchen Kontexten verwenden lässt, in denen eine neue Nuance hinzukommt.

In den Korpusdaten wird diese These bestätigt, und das sei hier anhand einiger Beispiele illustriert. So ist es etwa möglich, dass eine Frage mit denn eine tendenzielle Frage ist. Insbesondere die Nuance der Modalpartikel etwa, die auf eine negative Antwort ansteuert, kann bei denn impliziert sein. ${ }^{15}$ Diese 'etwa-Nuance' kann auch in der Übersetzung durchschimmern:

\footnotetext{
12 Damit ist aber keineswegs gemeint, diese Elemente seien völlig synonym. Sie werden nur dadurch vereint, dass sie diese Bedeutungsnuance zum Ausdruck bringen können; die anderen Bedeutungsaspekte sind für die hiesige Argumentation von geringerer Wichtigkeit und bleiben somit außer Betracht.

${ }^{13}$ Die Nummern I und II deuten nur auf die Reihenfolge, in der diese Verwendungen im Korpus vorgefunden wurden; es sind keine Annahmen zur Wichtigkeit oder zum historischen Verhältnis der Verwendungen impliziert.

${ }^{14}$ Es ist ohnehin ein gängiges Phänomen, dass Implikaturen im Laufe der Zeit Teil der Bedeutung werden, auch bei Grammatikalisierung.

${ }^{15}$ An sich ist denn tendenzneutral. Infolge der Erstaunensbedeutung, die gelegentlich mitschwingt, sollte aber die mögliche Implikation der 'etwa'-Nuance u. E. nicht allzu sehr wundern bzw. liegt sie mehr auf der Hand als die Implikation der Nuance von auch, mit der eine positive, bestätigende Tendenz angedeutet wird.
} 
(17) Bin ich denn des Wahnsinns? (bvn 116)

Je ne suis quand même pas folle? (117)

Hier erscheint in der Übersetzung ein quand même in Kombination mit einer Negation. Das ist die direkte Übersetzung des deutschen doch nicht, das von der Bedeutung her der Partikel etwa nahe steht. Diese Nuance, die hier bei denn implizit wirksam ist, zeigt sich somit ausdrücklich in der Übersetzung.

Ein zweites Beispiel wurde oben schon angesprochen. Es betrifft die Verwendung von vraiment als Ausdruck eines gewissen Unglaubens (= vraiment I). ${ }^{16}$ Diese Nuance des Unglaubens steht der des Erstaunens, die in denn gelegentlich mitschwingt (vgl. Kap. 1), ziemlich nahe. Somit sollte es nicht allzu sehr wundern, dass sie als zusätzlicher Bedeutungsaspekt der Partikel hinzukommen kann. Wie sich in Beispiel (16) zeigt, liegen solche Fälle tatsächlich vor, und es kann diese Nuance des Unglaubens durchaus in der Übersetzung expliziert werden, zum Beispiel mittels vraiment I:

(18) Muss das denn jetzt geschehen und gerade hier? (mjt 59)

Faut-il vraiment que cela se fasse maintenant, et de plus ici ? (58)

Der dritte hier zu besprechende Fall ist schon auffällig, da es sich um eine Bedeutungsnuance handelt, die normalerweise der Modalpartikel eigentlich zugeschrieben wird: das Markieren eines thematischen Übergangs. Wie sich in (17) zeigt, kann dieser Bedeutungsaspekt auch mit dem Gebrauch von denn in einer Frage einhergehen. Diese Erscheinung ist im Korpus viermal belegt. In der entsprechenden Übersetzung kann dieser Aspekt etwa mittels au fait expliziert werden:

(19) Woher kommt der denn? (her 28)

D'où vient-il, au fait ? (29)

In diesem Fall könnte man sich vielleicht fragen, inwiefern denn wirklich diesen thematischen Übergang bezeichnet bzw. ob dieser Bedeutungsaspekt im Deutschen nicht einfach unmarkiert bleibt. Wenn man davon ausgehen will, so müssten die vier Belege als zusätzliche Belege der Nichtübersetzung eingestuft werden. Dies würde aber die Verhältnisse zwischen den Partikeln weder in Kap. 6 noch in diesem Kapitel wesentlich ändern. Außerdem sind diese Beispiele in beiden Fällen als Belege einer fortgeschrittenen Grammatikalisierung zu analysieren (Bedeutungsverblassung bzw. neue Bedeutungserweiterung). Allerdings ist $\mathrm{u}$. $\mathrm{E}$. in diesem Fall die Nuance in der Partikel selber impliziert.

Es sei noch ein vierter Fall erwähnt, der von der Bedeutung her ebenfalls mit eigentlich zu verknüpfen ist, diesmal aber mit dem Adverb. Genauer gesagt liegt die Bedeutung von genau vor, und es ist dementsprechend das französische Pendant von genau, au juste, das in der Übersetzung erscheint:

(20) Wie viele hast du denn mitgenommen? (caf 170)

Tu en as pris combien, au juste ? (171)

Es dürfte also deutlich sein, dass bei denn verschiedene andere Nuancen impliziert sein können. Auch bei eigentlich ist das nicht unbedingt ausgeschlossen. Allerdings liegt im Korpus nur ein einziger Beleg vor:

(21) Wissen Sie eigentlich, was Sie sonst erwartet? (ste 326)

Savez-vous, réellement, ce qui vous attend autrement? (221)

Dieser Fall schließt sich dem mit vraiment I bei denn an: es liegt eine Unglaubensnuance vor, die in der Übersetzung mittels réellement I ausgedrückt wird. ${ }^{17}$

${ }^{16} \mathrm{Da}$ auch réellement I diese Bedeutung ausdrücken kann (vgl. (21)), ließe sich vermuten, dass auch dieses Adverb hier möglich gewesen wäre, und nur durch Zufall nicht belegt ist.

${ }^{17}$ In Kap. 1 wurde auch der Aspekt des thematischen Übergangs bei eigentlich als neue Bedeutungserweiterung gedeutet. Das ist zwar richtig, sollte hier aber nicht in Betracht gezogen werden, da diese Bedeutungserweiterung bei der Entwicklung zur Modalpartikel stattgefunden hat. In diesem Kapitel werden nur "spätere" Bedeutungserweiterungen in Betracht gezogen, d. h. Bedeutungserweiterungen, die erst stattgefunden haben, nachdem sich die betreffende Form schon als Modalpartikel etabliert hatte. Daher auch bleiben Übersetzungsäquivalente, die 
Es zeigt sich somit, dass sowohl denn als auch eigentlich andere Nuancen in sich aufnehmen können. Bei denn ist diese Tendenz aber stärker als bei eigentlich (es gibt mehr Belege bei denn, die außerdem eine größere Vielfalt aufweisen). Das hängt mit einer größeren Bedeutungsverblassung und somit einer weiter fortgeschrittenen Grammatikalisierung bei denn zusammen.

Zum Schluss sei noch kurz die schon mehrfach angesprochene Analyse Molnárs (2002) in Betracht gezogen. Es wurde schon angedeutet, dass eine konsekutive Bedetungsnuance in der Modalpartikel denn sich auch in der Übersetzung widerspiegeln kann, etwa in der Verwendung von alors. Oben wurde dieses Phänomen als Indiz der Bedeutungspersistenz gewertet. Nach der Analyse Molnárs müsste dies aber als neue Bedeutungserweiterung eingestuft werden. Diese Analyse ist u. E. weniger wahrscheinlich, würde aber die hiesige Argumentation nicht unbedingt schwächen, sondern eher verstärken. Das würde nämlich heißen, dass bei denn überhaupt keine Fälle vorliegen, bei denen die Persistenz in der Übersetzung durchschimmert. Dafür gäbe es dann aber zusätzliche Fälle, die mit neuer Bedeutungserweiterung zu verknüpfen sind, was sowohl die Anzahl als auch die Vielfalt der Belege, die sich so erklären lassen, erhöhte. In dem Sinne würde diese Analyse somit ein noch stärkeres Indiz für die weiter fortgeschrittene Bedeutungsverblassung (und neue Bedeutungserweiterung) bei denn liefern. Trotzdem wird hier nicht von dieser Analyse ausgegangen, weil sie aus diachronischer Sicht (d.h. in Bezug auf die Entwicklung von denn) als wenig plausibel erscheint. Außerdem ändert die Wahl der Analyse nicht sehr viel an der Argumentation, da es sich um weniger als $2 \%$ der Belege handelt: die aufgedeckten Tendenzen, die hier als Argumente verwendet werden, bleiben sowieso dieselben, ob nun an Bedeutungspersistenz oder an neue Bedeutungserweiterung angeknüpft wird.

\section{Die Kombination denn eigentlich}

Das fünfte und letzte Argument der vorliegenden Auseinandersetzung hat mit der Kombination der beiden hier betrachteten Partikeln, denn und eigentlich, zu tun. Allerdings muss gesagt werden, dass diese Kombination im Korpus nur mit zehn Belegen vertreten ist, so dass dieses fünfte Argument nur als eine Hypothese präsentiert werden kann, die anhand eines größeren Korpus weiter verifiziert werden müsste.

Zunächst seien aber noch ein paar allgemeine Beobachtungen vermittelt. Was die belegten Übersetzungsäquivalente angeht, liegen keine großen Überraschungen vor: sämtliche Pendants sind entweder bereits bei einer der Einzelpartikeln vertreten oder sind leicht damit $\mathrm{zu}$ verbinden. Im letzteren Fall handelt es sich um Synonyme belegter Übersetzungen der Einzelpartikeln, oder aber um eine Kombination solcher Übersetzungen.

Daneben fällt auf, dass die Nichtübersetzung hier weniger stark vertreten ist als bei den Einzelpartikeln. Das dürfte damit zusammenhängen, dass die Kombination zweier Partikeln als eine prominente sprachliche Form mit einer relativ starken Modalisierungsfunktion erfahren wird, die somit in der Übersetzung häufiger expliziert wird. Allerdings ist es so, dass oft nur ein Element in der Übersetzung erscheint, das dann entweder neutral ist was die Beziehung zu den Einzelpartikeln betrifft, oder aber stärker mit einer der beiden verbunden ist. Diese Tendenz zeigt sich vor allem in den bedeutungsstärkeren Übersetzungen, die 5 der 10 Belege ausmachen. Daneben liegen drei Fälle mit donc vor (23), einer mit diable (24) und wie bereits gesagt einer mit Nichtübersetzung (22):

(22) Was ist denn eigentlich los mit dem Herrn Kratky? (mjt 119)

Que se passe-t-il avec M. Kratky? (114)

(23) Was stand denn eigentlich in dem Brief? (pro 125)

Qu'y avait-il donc dans la lettre ? (246)

(24) Wohin ist denn eigentlich meine Schwester verschwunden? (mjt 36)

Où diable est passée ma sœur? (37)

einfach mit der Modalpartikel eigentlich übereinstimmen, d. h. die einfach einen thematischen Übergang andeuten (etwa au fait und d'ailleurs), hier außer Betracht. 
Dabei sei bemerkt, dass die Nichtübersetzung und donc bei beiden Einzelpartikeln vertreten sind, während das verstärkende diable nur bei denn belegt ist.

Daneben liegt ein Fall vor, in dem zwei Übersetzungsweisen kombiniert werden:

(25) Wenn ich heute bedenke, wer denn eigentlich die Romanisten waren, so stelle ich fest, dass es die Summe von sehr vielen jemanden war, von denen mir keiner persönlich besonders viel bedeutete. (mar 114)

Aujourd'hui, quand je réfléchis à ce que pouvaient être, en fait, les romanistes, je me dis que c'était la somme d'un grand nombre de Quelqu'un dont, personnellement, aucun ne représentait grand-chose pour moi. (142)

Am deutlichsten zeigt sich hier das Element, das mit eigentlich zu verknüpfen ist: en fait. Daneben taucht das Hilfsverb pouvoir auf, das bei denn in manchen Fällen (u. a. mit bien) ein sekundäres, verstärkendes Phänomen in der Übersetzung ist.

Neben diesen sechs Fällen gibt es vier Belege, deren Übersetzung nur ein einziges bedeutungsstärkeres Element aufweist. Dabei fällt auf, dass alle diese Pendants eher mit eigentlich zu verknüpfen sind, und zwar handelt es sich um Elemente, die alle von Bedeutungspersistenz in der deutschen Partikel zeugen: exactement (zwei Belege) (26), au juste (27) und au fond (28). Die ersten zwei sind auch bei der Einzelpartikel eigentlich vertreten, au fond nicht, aber dieses Element steht den anderen "Persistenzübersetzungen" von der Bedeutung her sehr nahe (vor allen denjenigen, die keine 'genau'-Nuance implizieren, wie en fait; vgl. Nøjgaard 1992-1995: §252).

(26) Wie alt sind Sie denn eigentlich? (frü 61)

Quel âge avez-vous exactement? (85)

(27) Wie kam er denn eigentlich zu dieser Waffe? (mjt 56)

Comment s'était-il procuré cette arme, au juste? (55)

(28) Was hat unsereiner denn eigentlich getan? (and 96)

Qu'est-ce que j'ai fait, moi, au fond? (166)

Wenn der Grenzfall (25) kurz außer Betracht gelassen wird, lassen sich die Übersetzungen hier genauso wie bei den Einzelpartikeln in bedeutungsvagere, eher mit denn zu verknüpfende Pendants einerseits, und bedeutungsstärkere, eher mit eigentlich zu verknüpfende Pendants andererseits einteilen. Dieses Bild entspricht auch der Ausgangsthese, denn sei weiter grammatikalisiert als eigentlich, denn wie oben in den Kapiteln 5 und 6 schon angedeutet wurde, stehen die Verblassungsübersetzungen eher auf der Seite von denn, während die Persistenzübersetzungen eher auf der Seite von eigentlich stehen.

Allerdings liegen bei denn ebenfalls bedeutungsstärkere Übersetzungen vor, nicht nur die schon angesprochenen (alors, vraiment I, negiertes quand même usw.), sondern auch andere, wie bien, die im vorliegenden Aufsatz nicht eingehender besprochen werden können. Was dabei auffällt, ist, dass diese Pendants bei der Übersetzung der Partikelkombination nicht belegt sind: es liegt entweder eine nichtaussagekräftige Übersetzung oder eine Übersetzung von eigentlich vor. Das ließe vermuten, dass sich Übersetzer, wenn sie nicht einfach eine illokutive Verstärkung einsetzen wollen, eher an eigentlich orientieren. Daraus könnte man schließen, dass eigentlich einen größeren Beitrag zu der Gesamtbedeutung der Partikelkombination liefert als denn.

Hier tritt allerdings ein Problem auf. Thurmair (1989: 204) behauptet nämlich, die Bedeutung von Partikelkombinationen sei rein kompositionell zusammengestellt. Für denn eigentlich gibt sie dazu einige Einschränkungen an. Einerseits darf mit eigentlich kein allzu drastischer Themenwechsel einhergehen, sondern nur ein Übergang zu einem anderen Aspekt desselben Themas. Sonst ließe sich die Kombination nicht mit der direkten Kontextanknüpfung von denn versöhnen. Andererseits darf bei denn keine allzu starke Nuance des Erstaunens o. ä. mitschwingen, weil sonst die Kombination mit dem thematischen Übergang nicht möglich wäre.

Problematisch ist dabei vor allem die Einschränkung, die denn auf eigentlich ausübt (eine Analyse, die von Lemnitzer 2001 bestätigt wird). Wenn man sich den Kontext von Beispielen 
wie (27) etwas genauer anschaut, fällt auf, dass doch ein ziemlich drastischer Themenwechsel vorliegt:

(27b) "Sollte dennoch das sein letzter Gedanke gewesen sein? Dieses Märchen für gläubige Kinder?" fragte der Ingenieur und sah mich unverwandt an.

Ich sagte nichts, ich wusste nicht, wovon er sprach. Er erwartete wohl auch keine Antwort.

"Never mind", sagte er zu sich selbst mit einer leichten Handbewegung. "Auch eines von den Dingen, denen wir nie auf den Grund kommen werden."

Er nahm den Revolver vom Tisch und sah ihn an mit einem Blick, der erkennen ließ, dass er an etwas anderes dachte. Dann legte er ihn wieder aus der Hand.

"Wie kam er denn eigentlich zu dieser Waffe?" fragte ich. (mjt 56)

Der Revolver, von dem in der Frage die Rede ist, wurde zwar im Roman gerade schon erwähnt, aber im Gespräch war vor der Frage von ganz Anderem die Rede. Es handelt sich hier somit u. E. nicht einfach um einen Übergang zu einem anderen Aspekt des Themas.

An und für sich sollte dies nicht allzu sehr wundern. Es wurde nämlich schon gezeigt, dass denn schon ein beträchtliche Strecke auf dem Weg der Grammatikalisierung und der damit einhergehenden Bedeutungsverblassung zurückgelegt hat. Es wäre demgemäß zu erwarten, dass dies nicht nur für die Einzelpartikel gilt, sondern auch in einer Kombination. Das würde also heißen, dass denn auch hier kaum noch eine Bedeutung hat ${ }^{18}$, und somit der anderen Partikel (in diesem Fall eigentlich) kaum noch irgendwelche Einschränkungen auferlegt. Demzufolge leistet eigentlich einen größeren Beitrag zur Bedeutung der Kombination, womit einerseits erklärt wird, dass es Fälle wie (27b) gibt, und andererseits, dass bei der Übersetzung der Kombination eigentlich offenbar das größere Gewicht hat im Vergleich zu denn. Allerdings sei abermals bemerkt, dass für die Kombination denn eigentlich im Korpus nur zehn Belege vorliegen und dass diese Beobachtungen zu dem relativ größeren Beitrag von eigentlich weitaus breiterer empirischer Validierung bedürfen.

\section{Schlussfolgerungen}

Die Distribution der verschiedenen Übersetzungen kann anhand verschiedener Parameter gedeutet werden. Im vorliegenden Beitrag wurden die Bedeutungsebenen des Grammatikalisierungsprozesses (Bedeutungsverblassung, Bedeutungspersistenz und neue Bedeutungserweiterung) in Betracht gezogen. Anhand der Ergebnisse einer Korpusstudie wurde argumentiert, dass denn bereits weiter grammatikalisiert ist als eigentlich. Dazu wurden fünf Argumente geliefert, die jeweils die Übersetzungsäquivalente mit einem Aspekt des Grammatikalisierungsprozesses in Beziehung setzen. Diese fünf Argumente seien hier nochmal kurz aufgereiht:

a) bei denn liegt eine größere Vielfalt an Übersetzungen vor als bei eigentlich;

b) bei denn sind die bedeutungsvagen bzw. bedeutungsleeren Äquivalente stärker vertreten als bei eigentlich;

c) bei eigentlich spielt die Bedeutungspersistenz eine größere Rolle als bei denn;

d) denn weist eine stärkere Bedeutungserweiterung (relativ mehr Belege sowie eine gröBere Vielfalt an neuen Nuancen) auf als eigentlich;

e) (Hypothese:) eigentlich liefert einen größeren Beitrag zur Bedeutung der Partikelkombination denn eigentlich als denn.

Abbildung 2 zeigt die drei Argumente (b, c, d), die direkt mit den Bedeutungsaspekten der Grammatikalisierung der Einzelpartikeln verknüpft wurden:

\footnotetext{
${ }^{18}$ Es sei hier angemerkt, dass Fälle, in denen denn noch eine stärkere Bedeutung hat, nicht ausgeschlossen sind. Bei der Einzelpartikel ist das auch nicht der Fall, wie u. a. in Kap. 6 gezeigt wurde. Ziel ist hier vor allem, zu zeigen, dass es nicht nur solche Fälle mit stärker einschränkendem denn gibt.
} 




Abb. 2: Die prozentmäßige Distribution der vorliegenden Übersetzungen, gruppiert nach Bedeutungsaspekt der Grammatikalisierung

Vor allem bei den zwei ersten Aspekten (Bedeutungsverblassung bzw. -persistenz) treten die Tendenzen deutlich hervor. Wie dieses Bild auch zeigt, ist es nicht so, dass die "Persistenzübersetzungen" bei eigentlich insgesamt stärker vertreten sind als die "Verblassungsübersetzungen". Das war auch nicht dasjenige, das gezeigt werden sollte. Wichtiger ist der beträchtliche Unterschied zur Distribution bei denn. Bei der neuen Bedeutungserweiterung tritt kein deutlicher Unterschied hervor, da es sich bei den beiden Partikeln um ganz wenige Belege handelt. ${ }^{19}$ Der Unterschied ist also nicht sehr groß, aber immerhin anwesend, und wichtig ist auf dieser Ebene vor allem die Vielfalt an neuen Nuancen, die bei denn durchaus größer ist als bei eigentlich. Dies lässt sich jedoch nicht in Abbildung 2 nachweisen.

Am Anfang des vorliegenden Aufsatzes wurde bereits angedeutet, dass die genaue Bedeutung der Modalpartikeln in großem Maße kontextabhängig ist und dass somit ein breites Spektrum an Übersetzungsäquivalente zur Verfügung steht. Dies hat sich in den besprochenen Beispielen deutlich gezeigt. Dabei sei nochmals darauf hingewiesen, dass die Vielfalt in Wirklichkeit noch größer ist als was hier dargelegt wurde (vgl. Abb. 1). So gibt es bei eigentlich durchaus auch Pendants, die einfach den thematischen Übergang andeuten (etwa au fait und d'ailleurs). Darauf wurde jedoch nicht weiter eingegangen, weil sie für die hiesige Argumentation eine geringere Bedeutung haben (vgl. Fussnote 19).

Es sei zum Schluss eingeräumt, dass bei der Analyse von Übersetzungen immer mit der Vermittlung durch einen Übersetzer bzw. der Interpretation des Übersetzers zu rechnen ist. Dementsprechend bietet die Methodologie der Übersetzungsanalyse 'nur' einen indirekten Zugriff auf das Forschungsobjekt: die semantische Struktur deutscher Modalpartikeln. Immerhin hat sich der methodologische Umweg über die französische Übersetzung als besonders vorteilhaft erwiesen. Aus dieser zusätzlichen Perspektive haben sich für die Analyse fünf Argumente ergeben, die ein starkes Indiz dafür sind, dass die Modalpartikel eigentlich noch eine stärkere und deutlicher abgrenzbare Bedeutung aufweist als denn, das bereits weiter grammatikalisiert ist. Diese Annahme ist an und für sich nicht neu (vgl. u. a. schon die Darstellung in Thurmair 1989), wird aber von unseren Korpusdaten unter Bezugnahme auf systematisch wiederkehrende Übersetzungsäquivalente empirisch validiert.

19 Da denn viel mehr Belege hat als eigentlich, verschwindet der große Unterschied der absoluten Belegzahlen dieser Gruppe zum Teil bei den relativen Zahlen. 


\section{Literatur}

Abraham, Werner (2000): "Modal particles in German: Word classification and legacy beyond grammaticalisation". In: Vogel, Petra M./Comrie, Bernard (eds.): Approaches to the Typology of Word Classes. Berlin/New York, Mouton de Gruyter: 321-350.

Albrecht, Jörn (1976): "Les équivalents de l'allemand eigentlich dans les dictionnaires bilingues et dans la réalité de l'usage". Cahiers de lexicologie 28: 60-73.

Albrecht, Jörn (1977): "Wie übersetzt man eigentlich eigentlich?" In: Weydt, Harald (ed.): Aspekte der Modalpartikeln. Studien zur deutschen Abtönung. Tübingen, Max Niemeyer: 19-37.

Auer, Peter/Günthner, Susanne (2003): "Die Entstehung von Diskursmarkern im Deutschen ein Fall von Grammatikalisierung?" Interaction and Linguistic Structures (InList) 38. Online verfügbar unter http://www.uni-potsdam.de/u/inlist/issues/38/Inlist38.pdf.

Autenrieth, Tanja (2002): Heterosemie und Grammatikalisierung bei Modalpartikeln. Eine synchrone und diachrone Studie anhand von eben, halt, e(cher)t, einfach, schlicht und glatt. Tübingen: Max Niemeyer. (= Linguistische Arbeiten 450).

Autenrieth, Tanja (2005): "Grammatikalisierung bei Modalpartikeln. Das Beispiel eben". In: Leuschner, Torsten/Mortelmans, Tanja/de Groodt, Sarah (eds.): Grammatikalisierung im Deutschen. Berlin, Walter de Gruyter: 309-334. (= Linguistik - Impulse \& Tendenzen 9).

Burkhardt, Armin (1995): "Zur Übersetzbarkeit von Abtönungspartikeln. Am Beispiel von Hofmannsthals Der Schwierige". Zeitschrift für germanistische Linguistik 23/2: 172-201.

De Mulder, Walter (2001): "La linguistique diachronique, les études sur la grammaticalisation et la sémantique du prototype : présentation". Langue française 130: 9-32.

Diewald, Gabriele (2007): "Abtönungspartikel". In: Hoffmann, Ludger (ed.): Handbuch der deutschen Wortarten. Berlin, Walter de Gruyter: 117-141.

Feyrer, Cornelia (1998): Modalität in Kontrast. Ein Beitrag zur übersetzungsorientierten Modalpartikelforschung anhand des Deutschen und des Französischen. Frankfurt am Main: Peter Lang.

Franck, Dorothea (1980): Grammatik und Konversation. Königstein im Taunus: Scriptor.

Geeraerts, Dirk/Grondelaers, Stefan/Speelman, Dirk (1999): Convergentie en divergentie in de Nederlandse woordenschat. Een onderzoek naar kleding- en voetbaltermen. Amsterdam: Meertens Instituut.

Heine, Bernd (2003): "Grammaticalization". In: Joseph, Brian Daniel/Janda, Richard D. (eds): The Handbook of Historical Linguistics. Oxford, Blackwell: 575-601.

Hentschel, Elke/Weydt, Harald (1983): "Der pragmatische Mechanismus: denn und eigentlich". In: Weydt, Harald (ed.): Partikeln und Interaktion. Tübingen, Max Niemeyer: 263273.

Hopper, Paul J. (1991): "On some principles of grammaticization". In: Traugott, Elizabeth Closs/Heine, Bernd (eds.): Approaches to Grammaticalization. Vol.I: Focus on Theoretical and Methodological Issues. Amsterdam/Philadelphia: John Benjamins: 17-35.

Jakobson, Roman (1959): "On Linguistic Aspects of Translation". In: Bower, Reuben A. (ed.): On Translation. Cambridge, Mass., Harvard University Press: 232-239.

Lehmann, Christian (2002): Thoughts on grammaticalization. Erfurt: ASSidUE.

Lemnitzer, Lothar (2001): "'Wann kommt er denn nun endlich zur Sache?' Modalpartikelkombinationen - eine korpusbasierte Untersuchung". In: Lehr, Andrea et al. (eds.): Sprache im Alltag. Berlin/New York, Walter de Gruyter: 349-371.

Lerot, Jacques (2001): "L'expression des fonctions discursives en français et le problème de la traductibilité des particules modales allemandes et néerlandaises". In: Beheydt, Ludo et al. (eds): Contrastief onderzoek Nederlands-Frans. Recherches contrastives néerlandaisfrançais. Louvain-la-Neuve, Peeters: 125-141.

Liefländer-Koistinen, Luise (1988): "Zur Funktion von Modalpartikeln in literarischen Texten". Neuphilologische Mitteilungen 89/4: 567-572.

Métrich, René (1997) : "De la non traduction des 'mots de la communication' de l'allemand en français". Zeitschrift für französische Sprache und Literatur 107/2: 143-172.

Métrich, René (2000): "Cinq traducteurs en quête d'équivalents. Remarques sur la traduction des particules modales dans Franz Kafka, La Métamorphose". In: Gréciano, Gertrud (ed.): Micro- et macrolexèmes et leur figement discursif. Leuven, Peeters: 299-313. 
Métrich, René/Faucher, Eugène/Courdier, Gilbert (1999): Les Invariables Difficiles. Dictionnaire allemand-français des particules, connecteurs, interjections et autres 'mots de la communication'. Tome 2: bald - geradezu. Nancy: Association des Nouveaux Cahiers d'Allemand.

Molnár, Anna (2002): Die Grammatikalisierung deutscher Modalpartikeln. Fallstudien. Frankfurt am Main: Peter Lang. (= MetaLinguistica 12).

Molnár, Anna (2008): "Pragmatische Sprachphänomene und das Grammatikalisierungskonzept". Argumentum 4: 280-289.

Mosegaard Hansen, Maj-Britt (1997): "Alors and donc in spoken French: A reanalysis". Journal of Pragmatics 28/2: 153-187.

Mosegaard-Hansen, Maj-Britt (1998): The Function of Discourse Particles. A study with special reference to spoken standard French. Amsterdam/Philadelphia: John Benjamins.

Nøjgaard, Morten (1992-1995): Les adverbes français. Essai de description fonctionnelle. København: Munksgaard.

Rinas, Karsten (2006): Die Abtönungspartikeln doch und ja: Semantik, Idiomatisierung, Kombinationen, tschechische Äquivalente. Frankfurt am Main: Peter Lang.

Thurmair, Maria (1989): Modalpartikeln und ihre Kombinationen. Tübingen: Max Niemeyer. (= Linguistische Arbeiten 223).

Traugott, Elizabeth Closs (1982): "From Propositional to Textual and Expressive Meanings: Some Semantic-Pragmatic Aspects of Grammaticalization". In: Lehmann, Winfred P./Malkiel, Yakov (eds): Perspectives on Historical Linguistics. Amsterdam/Philadelphia, John Benjamins: 245-271.

Traugott, Elizabeth Closs (1995): "Subjectification in Grammaticalisation". In: Stein, Dieter/Wright, Susan (eds): Subjectivity and Subjectivisation. Cambridge, Cambridge University Press: $31-54$.

Waltereit, Richard (2006): Abtönung: Zur Pragmatik und historischen Semantik von Modalpartikeln und ihren funktionalen Äquivalenten in romanischen Sprachen. Tübingen: Max Niemeyer.

Wegener, Heide (2002): "The evolution of the German modal particle denn". In: Wischer, Ilse/Diewald, Gabriele (eds.): New Reflections on Grammaticalization. Amsterdam/Philadelphia, John Benjamins: 379-394.

Weydt, Harald (1969): Abtönungspartikel. Die deutschen Modalwörter und ihre französischen Entsprechungen. Bad Homburg: Max Gehlen.

Zifonun, Gisela et al. (1997): Grammatik der deutschen Sprache. Berlin/New York: Walter de Gruyter. (= Schriften des Instituts für deutsche Sprache 7).

Zimmermann, Klaus (1981): "Warum sind die Modalpartikeln ein Lernproblem?" In: Weydt, Harald (ed.): Partikeln und Deutschunterricht. Abtönungspartikeln für Lerner des Deutschen. Heidelberg, Julius Groos: 111-122.

\section{Primärtexte}

ali Dirk Dobbrow (2004): Alina westwärts - Alina au loin. Toulouse: Presses

Universitaires du Mirail.

and Max Frisch (1999): Andorra. Stück in zwölf Bildern. Frankfurt am Main: Suhrkamp.

Max Frisch (1965): Andorra. Pièce en douze tableaux. Paris: Gallimard.

aut Ulrike Syha (2005): Autofahren in Deutschland - Conduire en Allemagne. Toulouse: Presses Universitaires du Mirail.

bvn Rebekka Kricheldorf (2006): Die Ballade vom Nadelbaumkiller - La Ballade du tueur de conifères. Toulouse: Presses Universitaires du Mirail.

caf Moritz Rinke (2007): Café Umberto - Café Umberto. Toulouse: Presses Universitaires du Mirail.

dsr Leo Perutz (2005): Der schwedische Reiter. München: DTV.

Leo Perutz (1987): Le Cavalier suédois. Paris: Phébus.

eis Falk Richter (2006): Unter Eis - Sous la glace. Toulouse: Presses Universitaires du Mirail.

ele Hugo von Hofmannsthal (2002): Électre-Elektra. Le Chevalier à la Rose-Der Rosenkavalier. Ariane à Naxos - Ariadne auf Naxos. Paris: GF Flammarion. 
frü Frank Wedekind (1997): Frühlings Erwachen. Eine Kindertragödie. München:

Goldmann.

Frank Wedekind (1974): L'Eveil du printemps. Tragédie enfantine. Paris: Gallimard.

her Sibylle Berg (2004): Herr Mautz - Monsieur M. Toulouse: Presses Universitaires du Mirail.

hit Ingrid Noll (1993): Der Hahn ist tot. Zürich: Diogenes.

Ingrid Noll (1996): Rien que pour moi. Paris: Calmann-Lévy.

kon Kerstin Specht (2003): Königinnendramen - Trois Reines. Toulouse: Presses

Universitaires du Mirail.

ljw Johann Wolfgang Goethe (1973): Die Leiden des Jungen Werther. Frankfurt am Main: Insel.

Johann Wolfgang Goethe (1947/1959): Les Souffrances du jeune Werther. Paris: Gallimard.

mar Fritz Zorn (1994/2006): Mars. Frankfurt am Main: Fischer.

Fritz Zorn (1979): Mars. Paris: Gallimard.

mjt Leo Perutz (2006): Der Meister des Jüngsten Tages. München: DTV.

Leo Perutz (1989): Le Maître du Jugement Dernier. Paris: Fayard - Le Livre de poche.

mlu Kerstin Specht (2005): Marieluise - Marieluise. Toulouse: Presses Universitaires du Mirail.

pro Franz Kafka (1979): Der Prozess. Frankfurt am Main: Fischer.

Franz Kafka (1957): Le Procès. Paris: Gallimard.

sch Stefan Zweig (1974/2007): Schachnovelle. Frankfurt am Main: Fischer.

Stefan Zweig (1991): Le Joueur d'échecs. Paris: Librairie Générale Française - Le Livre de Poche.

sid Hermann Hesse (1974): Siddhartha. Eine indische Dichtung. Frankfurt am Main: Suhrkamp.

Hermann Hesse (1925): Siddhartha. Paris: Grasset.

ste Rolf Hochhuth (1967/2006): Der Stellvertreter. Reinbek: Rowohlt.

Rolf Hochhuth (1963): Le Vicaire. Paris: Seuil.

ver Franz Kafka (1988): Die Verwandlung - La Métamorphose. Paris: Librairie Générale Française - Le Livre de poche. 